\section{Ambulante Ösophagusdilatation praktikabel}

\author{
Die Endoskopie mit dünnkalibrigen Geräten ermöglicht auch in der \\ HNO-Ambulanz die Ösophagusinspektion. Sogar die Ballondilatation der \\ Speiseröhre in Lokalanästhesie ist möglich, was die Komplikationsrate \\ deutlich reduziert.
}

M orbidität und Mortalität der Ösophagus-Endoskopie resultieren vor allem aus kardiopulmonalen Komplikationen der Allgemeinanästhesie. Oft ist die aber gar nicht nötig. So wir eine endoskopische Ballondilatation auch bei topischer Anästhesie oder Sedierung gut toleriert. Darauf weist eine retrospektive Auswertung von 38 Patienten mit insgesamt 54 Prozeduren zur Erweiterung des Ösophagus hin, von denen 20 Prozeduren nur mit topischer Anästhesie durchgeführt werden konnten. Als Endoskop verwendeten die Autoren ein transnasales Ösophagoskop mit einem Arbeitskanal von $2 \mathrm{~mm}$. Die verwendeten Ballone hatten eine Länge von $5 \mathrm{~cm}$ und ließen sich auf einen Durchmesser von $8 \mathrm{~mm}, 9 \mathrm{~mm}$ oder $10 \mathrm{~mm}$ aufblasen.

Das Alter der Patienten reichte von 13 bis 88 Jahren. $18 \%$ der Patienten waren laryngektomiert, $39 \%$ waren wegen eines Kopf-Hals-Tumors bestrahlt worden. Am häufigsten (63\%) musste die Speiseröhre im Bereich des oberen Ösophagussphinkters dilatiert werden gefolgt vom mittleren und unteren Ösophagussphinkter (26\% und 7,6\%) oder sowohl dem oberen und unteren Sphinkter (3,7\%). Die Indikation zu der Prozedur stellten die Ärzte aufgrund einer krikopharyngealen Dysfunktion, einer benignen Striktur oder einem Schatzki-Ring. Wenn eine topische Anästhesie nicht ausreichte, erfolgte eine Sedierung bei Bewusstsein.

Wesentliche Komplikationen fanden die Autoren in den Krankenakten nicht.
Kein Patient blieb anschließend in stationärer Behandlung. In zwei Fällen musste die Ösophagusendoskopie abgebrochen werden, einmal wegen eines Laryngospasmus, einmal wegen anhaltenden Würgens des Patienten.

fk

Rees CJ et al. Transnasal balloon dilation of the esophagus. Arch Otolaryngol Head

Neck Surg. 2009; 135: 781-3

\section{Kommentar}

Seit mehreren Jahren werden Ballon-Dilatationstechniken in verschieden Organbereichen eingesetzt. Für den HNO-Arzt ist besonders die Ballon-Katheter-Technik der Nasennebenhöhlen gegenwärtig. Jetzt wurde an einer relativ kleinen Patientengruppe versucht, die Vorteile einer Ballondilatation bei Ösophagusstenosen einzusetzen, wie sie nach Laryngektomien oder nach Bestrahlungen, aber auch nach Verätzungen auftreten. Die transnasal vorgeschobenen Katheter erlaubten eine für die Patienten wenig irritierende Manipulation unter Lokalanästhesie-Bedingungen. Zusätzlich konnte der jeweilige Dilatationseffekt unmittelbar endoskopisch kontrolliert werden. Offenbar gelang die Sondierung selbst sehr hochgradiger Stenosen mit einem Führungsdraht, was eine anschließend schonende Erweiterung der Stenose mit dem Ballon erlaubte. Obgleich der obere Ösophagusabschnitt die am häufigsten dilatierte Region war, konnten auch die mittleren und unteren Abschnitte ein- schließlich des unteren Sphinkters dilatiert werden.

Die einfach erscheinende Technik könnte nach Sammlung weiterer klinischer Erfahrungen ein relevantes Verfahren zur Behandlung von Ösophagusstenosen darstellen. Der zu diskutierende und derzeit nicht gelöste Nachteil ist die fehlende Kontrolle über die genaue Positionierung des Ballons und den Effekt der Dilatation. Hier wird vorgeschlagen, die sonst übliche Röntgendurchleuchtung durch eine endoskopische Kontrolle zu ersetzen. Inwieweit dies erreicht werden kann, hängt einerseits von den zur Verfügung stehenden Endoskopen und andererseits von den Erfahrungswerten des Anwenders ab. Eine gewisse Lernkurve dürfte dazu erforderlich sein. Eine weitere offene Frage ist die nach dem maximal dilatierbaren Lumen, je nach Stenoseursache und -befund. Vorteil des Verfahrens wäre in erster Linie, dass es sich schonend durchführen und einfach wiederholen ließe. Somit könnte die Methode nach Prüfung der klinischen Sicherheit Einzug in die Behandlungspraxis des HNOArztes nehmen, der schwerpunktmäßig onkologische Patienten betreut. Zunächst sollten in HNO-Kliniken in Zusammenarbeit mit Radiologen in klinischen Studien die Sicherheit der vorgestellten Technik geprüft werden. Weitere Fragen, die sich noch ergeben werden, sind die nach den Kosten und der Machbarkeit unter ambulanten oder stationären Bedingungen.

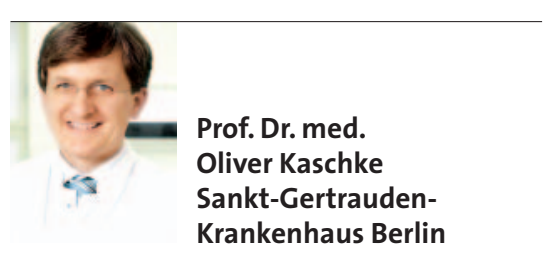

